\title{
Estimation of black carbon emissions from Siberian fires using satellite observations of absorption and extinction optical depths
}

Igor B. Konovalov et al.

Correspondence to: Igor B. Konovalov (konov@appl.sci-nnov.ru)

Here, we report several supplementary tests that were designed to evaluate the sensitivity of the empirical AAOD parameterization (see Eq. 4) discussed in Sect. 2.2.3 with respect to possible biases in the simulated AAOD and AOD data for the "bgr" scenario (see Eq. 5). The different test cases involved different $\mathrm{AAOD}_{\mathrm{b}}$ and $\mathrm{AOD}_{\mathrm{b}}$ values that were modified by applying to them the constant scaling factors, $s_{1}$ and $s_{2}$, respectively.

First, we considered the case where the both $s_{1}$ and $s_{2}$ were equal zero. That is, an impact of the "background" aerosol on the columnar SSA values was entirely disregarded. This case is analogous to one addressed in Konovalov et al. (2017a), except that the data selection criteria were different (see Sect. 2.2.3). The estimate of $\kappa_{1}$ obtained in this case (see Fig. S1) is $15 \%$ lower than that for the base case (see Fig. 4). The difference can be explained by the uncertainty at the $90^{\text {th }}$ percentile confidence level. Using the parameterization presented in Fig. S1 for estimation of BB BC emission estimation procedure would accordingly result in about 15 \% larger top-down estimates of BC emissions than those reported in Sect. 3.5 .

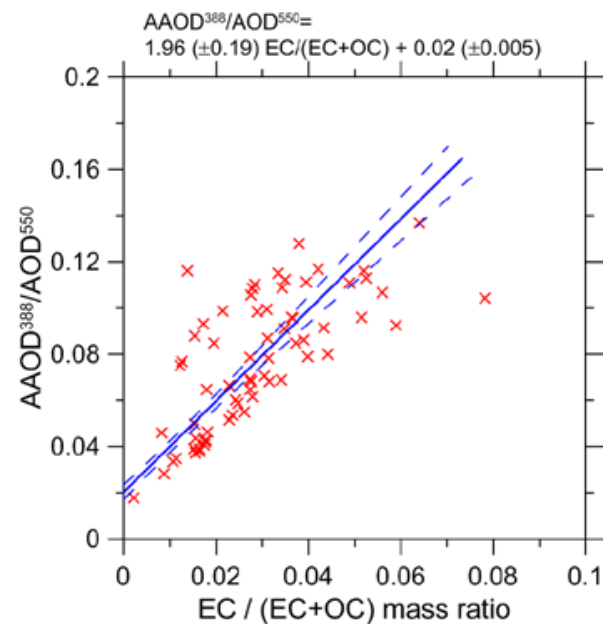

Figure S1: The AAOD/AOD ratio as a function of the $\mathrm{EC} /(\mathrm{EC}+\mathrm{OC})$ ratio. Both ratios shown by red crosses have been derived from the observations at the AERONET sites as explained in Sect. 2.2.3, except that the $\mathrm{AAOD}_{\mathrm{b}}$ and $\mathrm{AOD}_{\mathrm{b}}$ values involved in Eq. (5) were zeroed. A linear regression fitted with the ODR method and its $68.3 \%$ (1-sigma) confidence intervals are shown by solid and dashed blue lines, respectively.

In the second test, $s_{1}$ was equal to 0.5 or $1.5\left(\mathrm{AAOD}_{\mathrm{b}}\right.$ was increased or decreased by $\left.50 \%\right)$, while $s_{2}$ was equal to 1.0 . The results indicate (see Fig. S2) that the parameterization is quite insensitive to the large changes in the background AAOD values. Although we cannot estimate possible biases in $\mathrm{AAOD}_{\mathrm{b}}$, the fact that the decrease of the $\mathrm{AAOD}_{\mathrm{b}}$ values decreases the uncertainty of both $\kappa_{1}$ and $\kappa_{2}$ may be regarded as evidence that $\mathrm{AAOD}_{\mathrm{b}}$ is biased positively.

Finally, in the third test, the $\mathrm{AAOD}_{\mathrm{b}}$ values were kept constant ( $s_{1}$ was equal 1.0) but the $\mathrm{AOD}_{\mathrm{b}}$ values were increased or decreased by $50 \%$ ( $s_{2}$ was set to be 0.5 or 1.5). The decrease of $\mathrm{AOD}_{\mathrm{b}}$ results in a rather small decrease of $\kappa_{1}$ (by $8 \%$ ), but the increase of $\mathrm{AOD}_{\mathrm{b}}$ leads to a substantial increase (by $20 \%$ ) of the same coefficient. Therefore, if $\mathrm{AOD}_{\mathrm{b}}$ is strongly underestimated, the AAOD/AOD ratio may also be considerably underestimated in our simulations, while the BB BC emissions obtained in our analysis may be accordingly overestimated. However, although we do not have any information about probable biases in $\mathrm{AOD}_{\mathrm{b}}$ at the AERONET sites considered in this study, there is evidence (see Sect. 3.6) that $\mathrm{AOD}_{\mathrm{b}}$ is 
actually overestimated by about $40 \%$ on average for the whole study region. Therefore, the underestimation of AAOD/AOD ratio by the parameterization presented used in our analysis (see Fig. 4) is possible but not likely.

(a)

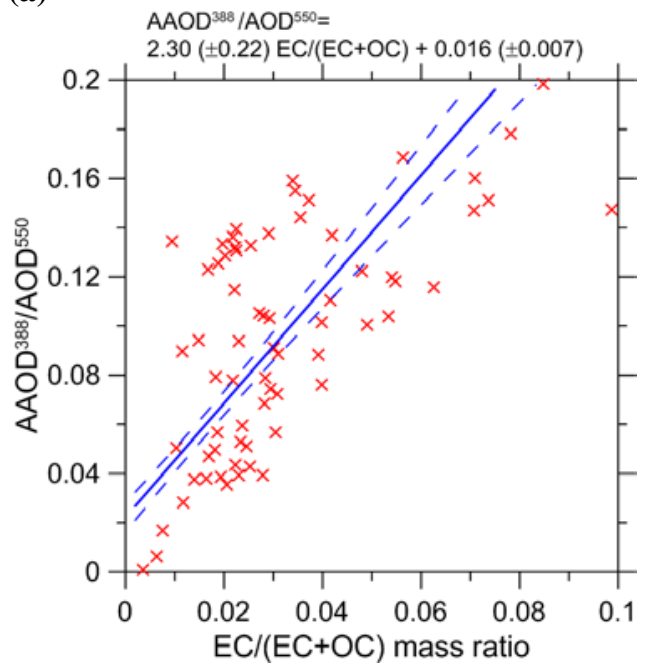

(b)

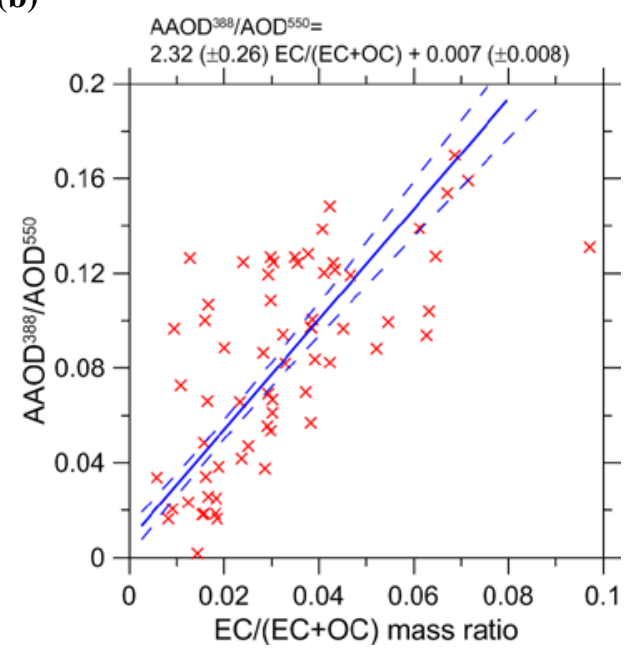

Figure S2: The same as in Fig. S1, but with the original $\mathrm{AOD}_{\mathrm{b}}$ values and with the $\mathrm{AAOD}_{\mathrm{b}}$ values (see Eq. (5)) that were (a) decreased by a factor 0.5 and (b) increased by a factor of 1.5 .

(a)

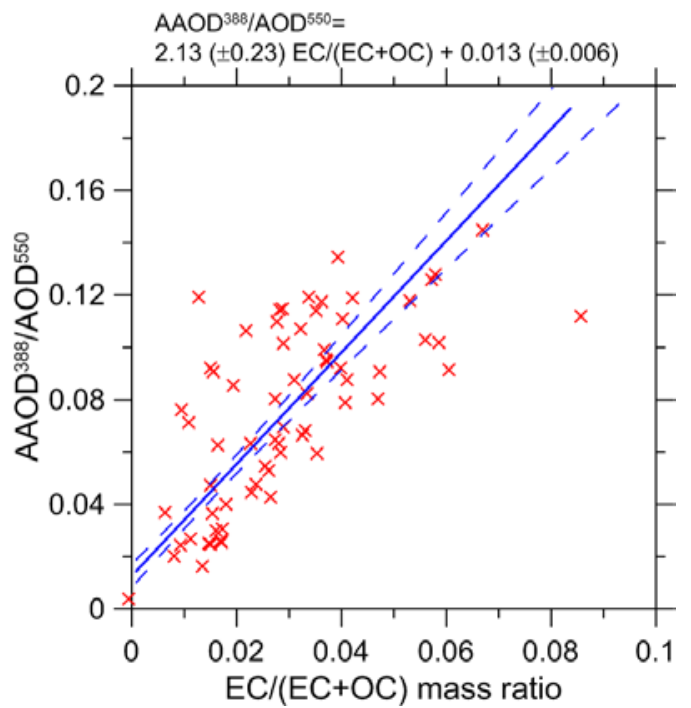

(b)

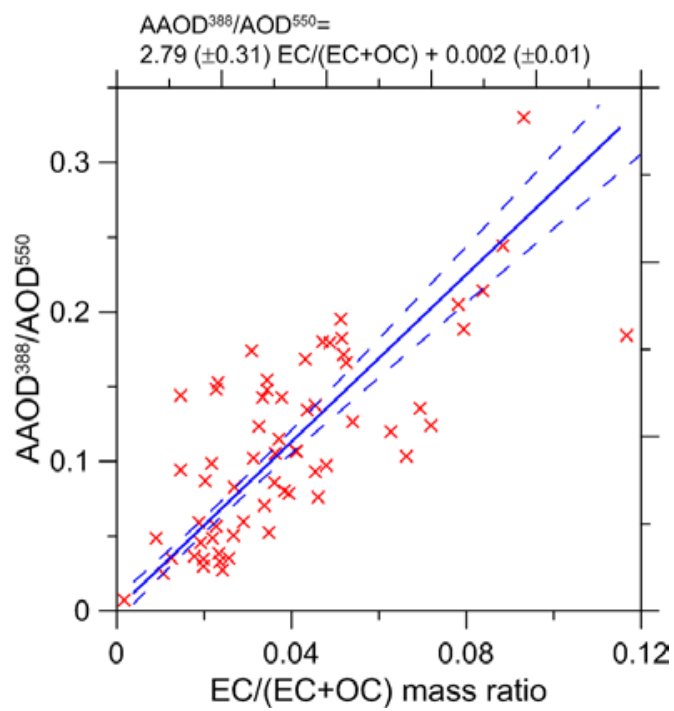

Figure S3: The same as in Figs. S1 and S2, but with the original $A_{A O D}$ values and with the AOD $_{b}$ values that were (a) decreased by a factor 0.5 and (b) increased by a factor of 1.5 . 